\title{
Dopaminergic neurons generated from monkey embryonic stem cells function in a Parkinson primate model
}

\author{
Yasushi Takagi, ${ }^{1,2}$ Jun Takahashi, ${ }^{1}$ Hidemoto Saiki, ${ }^{3}$ Asuka Morizane, ${ }^{1}$ Takuya Hayashi, ${ }^{4}$ Yo Kishi, ${ }^{1}$
} Hitoshi Fukuda, ${ }^{1}$ Yo Okamoto, ${ }^{1}$ Masaomi Koyanagi, ${ }^{1}$ Makoto Ideguchi, ${ }^{1}$ Hideki Hayashi, ${ }^{1}$ Takayuki Imazato, ${ }^{1}$ Hiroshi Kawasaki, ${ }^{5}$ Hirofumi Suemori, ${ }^{6}$ Shigeki Omachi, ${ }^{7}$ Hidehiko lida, ${ }^{4}$ Nobuyuki Itoh, ${ }^{7}$ Norio Nakatsuji, ${ }^{6}$ Yoshiki Sasai, ${ }^{2,5}$ and Nobuo Hashimoto'

\begin{abstract}
${ }^{1}$ Department of Neurosurgery, Kyoto University Graduate School of Medicine, Kyoto, Japan. ${ }^{2}$ rganogenesis and Neurogenesis Group, Center for Developmental Biology, RIKEN, Kobe, Japan. ${ }^{3}$ Department of Neurology, Kyoto University Graduate School of Medicine, Kyoto, Japan. ${ }^{4}$ Department of Experimental Radiology, National Cardiovascular Center, Osaka, Japan. ${ }^{5}$ Department of Medical Embryology and Neurobiology and ${ }^{6}$ Department of Development and Differentiation, Institute for Frontier Medical Sciences, Kyoto University, Kyoto, Japan. 7Department of Genetic Biochemistry, Kyoto University Graduate School of Pharmaceutical Sciences, Kyoto, Japan.
\end{abstract}

\begin{abstract}
Parkinson disease (PD) is a neurodegenerative disorder characterized by loss of midbrain dopaminergic (DA) neurons. ES cells are currently the most promising donor cell source for cell-replacement therapy in PD. We previously described a strong neuralizing activity present on the surface of stromal cells, named stromal cellderived inducing activity (SDIA). In this study, we generated neurospheres composed of neural progenitors from monkey ES cells, which are capable of producing large numbers of DA neurons. We demonstrated that FGF20, preferentially expressed in the substantia nigra, acts synergistically with FGF2 to increase the number of DA neurons in ES cell-derived neurospheres. We also analyzed the effect of transplantation of DA neurons generated from monkey ES cells into 1-methyl-4-phenyl-1,2,3,6-tetrahydropyridine-treated (MPTP-treated) monkeys, a primate model for PD. Behavioral studies and functional imaging revealed that the transplanted cells functioned as DA neurons and attenuated MPTP-induced neurological symptoms.
\end{abstract}

\section{Introduction}

Parkinson disease (PD) is a neurodegenerative disorder characterized by the loss of midbrain dopaminergic (DA) neurons, with subsequent reductions in striatal dopamine levels. While initial pharmacological treatment with L-dihydroxyphenylalanin (L-DOPA) can attenuate symptoms, the efficacy of this treatment gradually decreases over time. The development of motor complications then requires additional treatments, including deep brain stimulation and fetal DA neuron transplantation (1-3). Both studies of animal models and clinical investigations have shown that transplantation of fetal DA neurons can produce symptomatic relief (4-8). The technical and ethical difficulties in obtaining sufficient and appropriate donor fetal brain tissue, however, have limited the application of this therapy.

ES cells are self-renewing, pluripotent cells derived from the inner cell mass of the preimplantation blastocyst. These cells have many of the characteristics required of a cell source for cell-replacement therapy, including proliferation and differentiation capacities (9). We previously discovered that a strong neuralizing activity, which we called stromal cell-derived inducing activity (SDIA), is present

\footnotetext{
Nonstandard abbreviations used: ChAT, choline acetyltransferase; DA, dopaminergic; DAT, dopamine transporter; GABA, $\gamma$-amino butyric acid; GalC, galactocerebroside C; GFAP, glial fibrillary acidic protein; GMEM, Glasgow minimum essential medium; i.m., intramuscular(ly); LIF, leukemia inhibitory factor; ME, mercaptoethanol; MPTP, 1-methyl-4-phenyl-1,2,3,6-tetrahydropyridine; NCAM, N cell adhesion molecule; OL, poly-l-ornithine and laminin; PD, Parkinson disease; PET, positron emission tomography; SDIA, stromal cell-derived inducing activity; $\mathrm{TH}$, tyrosine hydroxylase. Conflict of interest: The authors have declared that no conflict of interest exists.

on the surface of stromal cells. In the absence of exogenous bone morphogenic protein-4, mouse ES cells differentiate efficiently into neural precursors and neurons when cultured for 1 week on SDIA-expressing mouse stromal cells (PA6 cells) (10). Recently, SDIA induction has also been applied to primate ES cells, which generated large numbers of neural precursors and postmitotic neurons when cultured on PA6 cells for two weeks (11). The SDIA method is both technically simple and efficient, producing significant numbers of midbrain DA neurons $(10,11)$.

Self-renewing, multipotent neural progenitors can be cultured as neurospheres (12). In this study, we generated neural progenitors from monkey ES cells, then expanded them as neurospheres, which contained progenitors of DA neurons. In addition, we analyzed the effect of FGF20, a novel member of the FGF family of growth factors that is expressed exclusively in the substantia nigra of the brain and is reported to have a protective effect on DA neurons (13). We observed increased DA neuron induction following treatment with FGF20. Furthermore, we transplanted neurons generated by this method into 1-methyl-4-phenyl-1,2,3,6tetrahydropyridine-treated (MPTP-treated) cynomolgus monkeys, a primate model for PD. We found that transplanted cells were able to function as DA neurons and could diminish Parkinsonian symptoms. This is the first report, to our knowledge, demonstrating the efficacy of transplantation therapy using ES cell-derived DA neurons in an experimental primate model of PD.

\section{Results}

Induction of neural progenitors from monkey ES cells. To enrich graftable neural progenitors, we first cultured monkey ES cells on PA6 stromal feeder cells, then detached the cells from the feeders for 
A

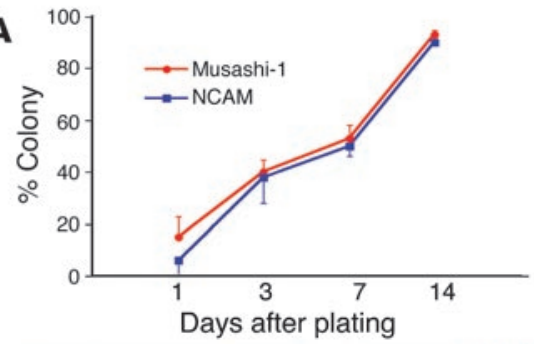

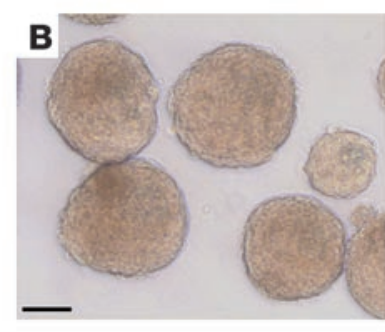

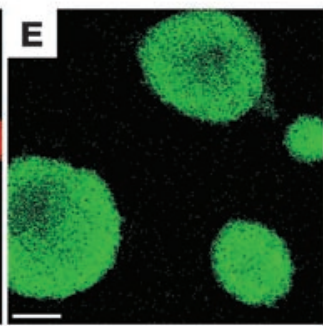

\section{Figure 1}

Neural progenitors induced from primate ES cells. (A) Time course of neural progenitor marker expression in monkey ES cells cultured on PA6 cells. (B) Detached ES cell colonies formed spheres similar to those of neural progenitor cells. (C-E) Spheres were immunoreactive for NCAM (C, green), Musashi-1 (D, red), and Nestin (E, green). Scale bar: $100 \mu \mathrm{m}$.

cells formed spheres morphologically resembling those formed by neural progenitor cells (Figure 1B). These spheres were positively stained with antibodies specific for neural progenitor cell markers NCAM, Musashi-1, and Nestin (Figure 1, C-E). To determine the potential of these cells to differentiate, we expanded the cells as spheres for 7 days, then induced differentiation by culturing the cells on poly-l-ornithine and laminin-coated (OL-coated) slides for 7 days. We removed the mitogens FGF2, EGF, and LIF from the medium, instead adding expansion as neurospheres. ES cells began to differentiate on PA6 cells, with cells immunoreactive for neural progenitor markers, such as N cell adhesion molecule (NCAM) and Musashi-1 $(11,14)$, emerging within three days. ES cells proliferated and differentiated on the feeder layer by forming colonies, and cells positive for neural markers increased in number until 2 weeks into the culture period. The percentages of the colonies including at least 1 NCAM- or Musashi-1-positive cell reached approximately $100 \%$ at 2 weeks of culture $(\mathrm{NCAM}=90.4 \% \pm 7.5 \%$, Musashi- $1=97.5 \% \pm 4.6 \%, n=100$ from 3 independent cultures; Figure 1A). At this time point, $78.3 \% \pm 7.5 \%$ of the total cells were immunoreactive for NCAM, $75.0 \% \pm 15.4 \%$ for Musashi- 1 , and $72.3 \% \pm 9.1 \%$ for another neural progenitor marker, Nestin (14). These results indicate that the majority of the ES cells were committed to the neural lineage by day 14 of culture on feeder layers.

In vitro characterization of neural progenitors derived from monkey ES cells. To further enrich neural progenitors, we detached the ES cells from the feeder layer on day 14 , then continued to culture the cells on noncoated dishes in serum-free medium containing FGF2, EGF, and leukemia inhibitory factor (LIF). During the next few days, the neurotrophic factors such as brain-derived neurotrophic factor and neurotrophin-3. Immunofluorescence analysis revealed that the cells differentiated into mature neural cells expressing the neuronal markers TuJ $1(52.8 \% \pm 16.0 \%$ of DAPI $)$ and Map2ab $(38.3 \% \pm 7.5 \%$ of DAPI), the astroglial marker glial fibrillary acidic protein (GFAP) $(28.6 \% \pm 17.6 \%$ of DAPI), and the oligodendroglial marker galactocerebroside C (GalC) $(0.6 \% \pm 0.4 \%$ of DAPI) (Figure $2, \mathrm{~A}-\mathrm{D}$ and I). Further analyses demonstrated that these neurons derived from ES cells were immunoreactive for $\gamma$-amino butyric acid (GABA) $(28.6 \% \pm 10.7 \%$ of TuJ 1$)$, glutamate $(14.3 \% \pm 5.3 \%$ of TuJ 1$)$, choline acetyltransferase (ChAT) $(0.7 \% \pm 0.3 \%$ of TuJ1), and serotonin $(3.3 \% \pm 1.7 \%$ of TuJ1) (Figure 2, E-H and J). These results suggest that ES cell-derived spheres contain neural progenitor cells.

Effect of growth factors on differentiation of DA neurons. Effective treatment of $\mathrm{PD}$ requires substantial quantities of DA neurons. The percentage of tyrosine hydroxylase-positive (TH-positive) cells derived from neurospheres was only $5.4 \% \pm 1.8 \%$ of the TuJ1-positive cells (Figure 2J). To increase the percentage of these cells, we examined the effects of various combinations of growth factors on neurosphere culture. The percentage of
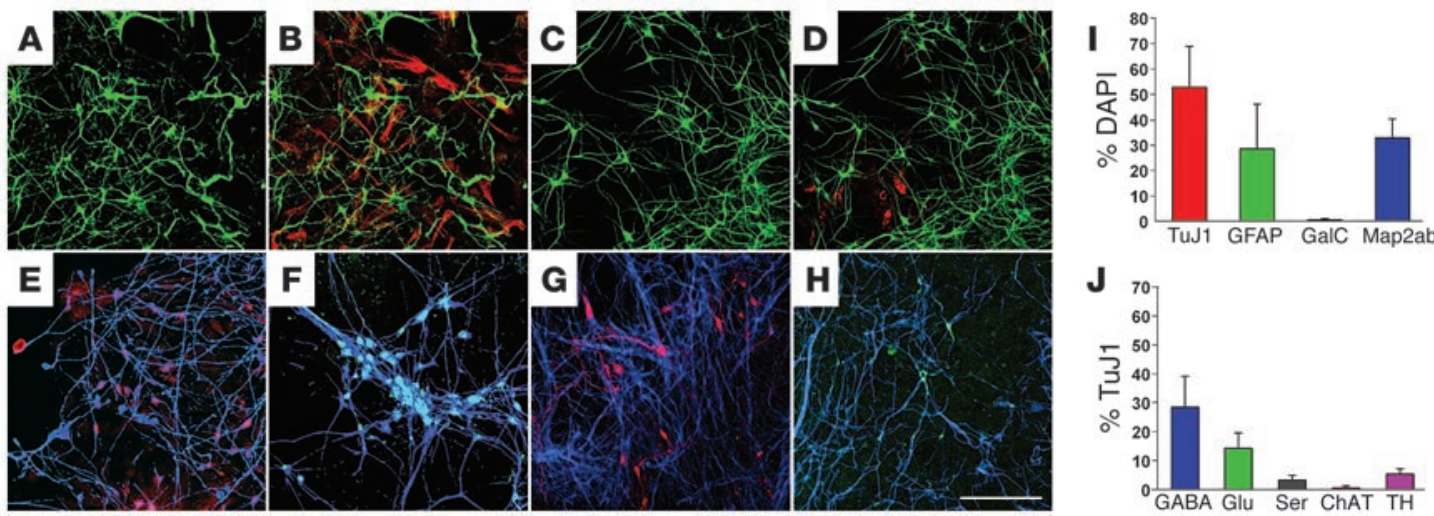

\section{Figure 2}

Expression of differentiated neural and neuronal subtype markers. Differentiated spheres were stained with antibodies against TuJ1 (A and B, green; E-H, blue), GFAP (B, red), Map2ab (C and D, green), GalC (D, red), GABA (E, red), glutamate (Glu; F, green), serotonin (Ser; G, red), and ChAT $(\mathbf{H}$, green). Scale bar: $100 \mu \mathrm{m}$. The proportions of cells expressing differentiated neural $(\mathbf{I})$ and neurotransmitter-related $(\mathbf{J})$ markers are expressed as the mean \pm SD of 3 independent cultures. 

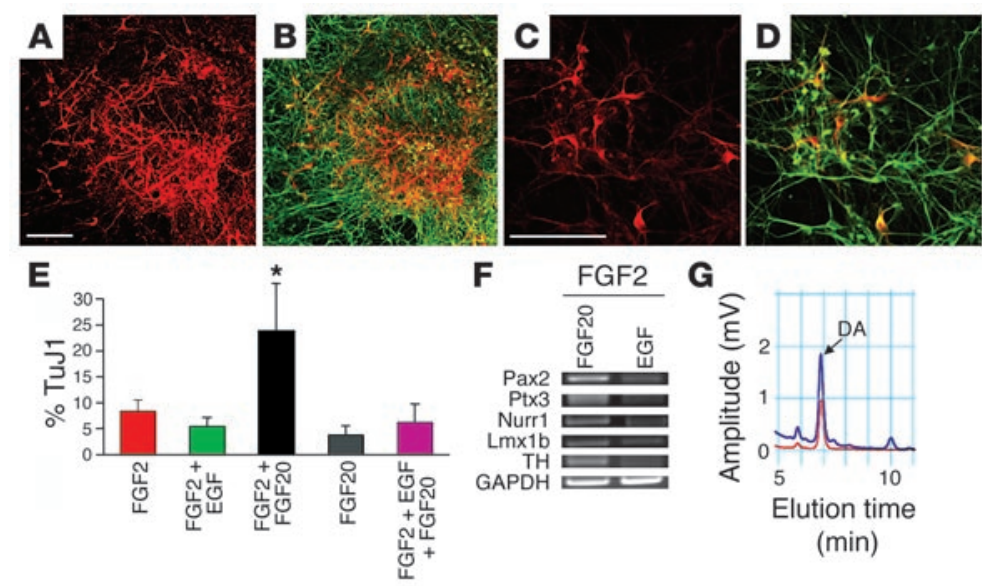

F

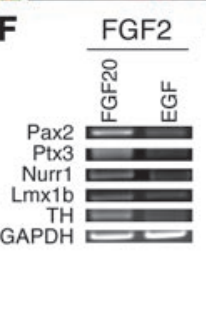

\section{G}

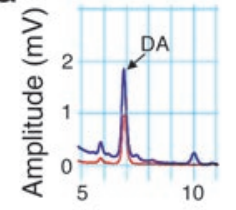

Elution time

(min)

\section{Figure 3}

DA neurons differentiated from ES cell-derived neurospheres. (A-D) Differentiated spheres treated with FGF2 and FGF20 were stained with antibodies against TH (red) and TuJ1 (green). Scale bars: $50 \mu \mathrm{m}$. (E) The proportion of TH-positive cells to TuJ1-positive cells is expressed as the mean \pm SD of 3 to 5 independent cultures. ${ }^{*} P<0.05$. (F) RT-PCR for mesencephalic DA neuron markers Pax2, Ptx3, Nurr1, Lmx1b, and $\mathrm{TH}$ in cells treated with FGF2 and FGF20 (left) or FGF2 and EGF (right). (G) HPLC measuring concentration of dopamine released by SDIA- and FGF2- and FGF20-treated monkey $\mathrm{ES}$ cells in response to high $\mathrm{K}^{+}$depolarizing stimuli (blue line). Dopamine standard, red line.
TH-positive neurons out of total Tuj1-positive cells increased to $24 \%$ in the presence of FGF2 and FGF20 (Figure 3, A-E). Neither FGF2 nor FGF20 alone caused an increase in the number of $\mathrm{TH}$-positive neurons. In the presence of FGF2, however, FGF20 increased the rate of $\mathrm{TH}$ differentiation in a dose-dependent manner $(1 \mathrm{pg} / \mathrm{ml}=3.8 \% \pm 1.8 \%$ of TuJ $1,10 \mathrm{pg} / \mathrm{ml}=8.8 \% \pm 4.8 \%$ of TuJ1, $1 \mathrm{ng} / \mathrm{ml}=24.3 \% \pm 9.8 \%$ of TuJ 1 ).

RT-PCR analyses showed that the cells derived from SDIAinduced neurospheres expressed mesencephalic DA neuron markers such as Pax2, Ptx 3, Nurr $1, \operatorname{Lm} \times 1$ b, and TH $(15,16)$. The expression of these markers was more abundant in FGF2- and FGF20-treated cells than in FGF2- and EGF-treated cells (Figure 3F). Furthermore, FGF2- and FGF20-treated cells released $55.6 \pm 19.9$ pmol per $10^{6}$ cells of dopamine in response to high $\mathrm{K}^{+}$ depolarizing stimuli as assayed by HPLC ( $n=5$; Figure $3 \mathrm{G})$. These results indicate that monkey ES spheres treated both with SDIA and with FGF2 and FGF20 generate a significant number of functional DA neurons in vitro.

Transplantation of DA neurons from ES-derived neural progenitors. To determine if the isolated TH-positive neurons function as DA neurons in vivo, ES cell-derived neurospheres were grafted into the putamen of the monkeys with MPTP-induced PD. In this primate model of PD, we administered MPTP intravenously to Macaca fascicularis (cynomolgus monkeys), hereafter $M$. fascicularis, and evaluated their behavior by scoring for neurological symptoms, such as motility and tremor (Table 1). Only the animals that exhibited stable deterioration for periods longer than 12 weeks were used for transplantation. ES cell-derived neurospheres used for grafting were prepared by treating with SDIA for 14 days and, subsequently, with FGF2 and FGF20 for 7 days, as described above. Using MRI images obtained for each monkey, we determined the necessary coordinates to stereotactically transplant the cells (300,000-600,000 cells per side) into the bilateral putamen. After injection, we continuously analyzed the behavior of postoperative monkeys by assessing neurological scores. We observed a slight recovery in the behavioral symptoms even in the sham-operated animals. At 10 weeks after transplantation, however, the mean scores of ES cell-transplanted monkeys significantly improved over the levels observed in sham-operated ones (Figure 4A, $n=6$ and 4, respectively). In our evaluation of symptoms after transplantation, posture recovery was the most prominent improvement seen in ES cell-transplanted monkeys, with significant improvements in motility also observed. There were, however, no significant changes in head-checking movement. Consciousness was not disturbed in any preoperative animals, and no deterioration was observed postoperatively. In addition, none of the treated animals developed dyskinesia. Positron emission tomography (PET) at 14 weeks after transplantation revealed increases in ${ }^{18} \mathrm{~F}$-fluorodopa uptake at the putamen of the ES cell-transplanted animals (Figure 4, B and C).

After the PET study, animals were sacrificed and subjected to immunohistochemical analysis. Grafted cells, which were labeled by BrdU treatment during sphere culture, were detected in the putamen of ES cell-transplanted monkeys (7,996 $\pm 3,300$ cells per side; Figure 5, A-C). DA neurons were detected by TH (Figure 5, B, C, E, and F; ref. 11) or dopamine transporter (DAT) (Figure 5, H and I; ref. 17) staining. An average of $2130 \pm 645$ TH-positive cells per side survived. Double labeling immunofluorescence microscopy revealed that $65.5 \% \pm 4.3 \%$ and $50.3 \% \pm 6.1 \%$ of the TH- and DAT-positive cells were immunoreactive for BrdU, respectively. Thus, the estimated number of TH/BrdU-double-positive cells was 1395 per side, $17.4 \%$ of the total of BrdU-positive cells. In contrast, in sham-operated monkeys, only a few TH-positive cells and scattered fibers were detectable in the putamen. No tumor formation or Ki-67-inducing reactivity was observed. As for other

\section{Table 1}

Neurological scores of MPTP-treated monkeys

Behavior
Alertness
Head-checking movement
Eyes
Posture
Balance
Motility, at rest
Motility, reaction to external stimuli
Walking
Tremor

\section{Scores}

Normal, 0; reduced, 1; absent, 2

Present, 0 ; reduced, 1 ; absent, 2

Normal, 0; reduced blinking, 1; eyes closed, 2

Normal, 0; mildly abnormal, 1; abnormal, 2; grossly abnormal, 3

Normal, 0; impaired, 1; frequent falling, 2; no movement, 3

Normal, 0; mild bradykinesia, 1; bradykinesia, 2; akinesia, 3

Normal, 0; mildly reduced, 1 ; reduced, 2; absent, 3

Normal, 0; mildly reduced, 1 ; reduced, 2; no walking, 3

Absent, 0; mild/not always, 1 ; moderate, 2; severe, 3 


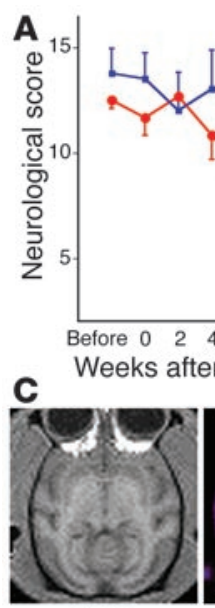

MRI

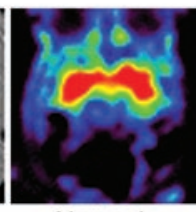

Normal
B

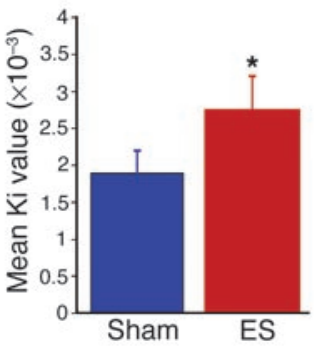

Figure 4

Function of ES cell-derived neurospheres in MPTP-treated monkeys. Behavioral scores $(\mathbf{A})$ and PET study $(\mathbf{B}$ and $\mathbf{C})$ of ES cell-transplanted $(n=6)$ and sham-operated animals $(n=4)$. (B) Mean Ki values from entire putamen. (C) Increased ${ }^{18} \mathrm{~F}$-fluorodopa uptake in the putamen of ES cell-transplanted animals. All values are mean \pm SD. ${ }^{*} P<0.05$.

neuronal phenotypes, GABA-positive cells were detected in the graft at a slightly higher frequency than TH-positive cells, while few serotonin-positive cells were present (data not shown).

\section{Discussion}

Following initial work by Thomson et al. reporting a method for establishing primate ES cells (18), Suemori et al. (19) recently devised a similar scheme for generating $M$. fascicularis ES cells. Lee et al. recently reported a 5-step method to induce DA neurons from ES cells through the induction of neural progenitor cells from embryoid bodies (20). Transplantation of induced DA neurons derived from mouse ES cells improves the neurological symptoms of rats with a Parkinson-like syndrome induced by treatment with 6-hydroxydopamine (6-OHDA) (21). We have also reported a method of inducing DA neurons based on SDIA resulting from coculture of ES cells on a PA6 stromal feeder layer. By the use of this method, mouse ES cells are diverted to a neuronal fate with TH-positive DA neurons composing $30 \%$ of total TuJ1-positive neurons (10). Furthermore, this method produced similar results with $M$. fascicularis ES cells (11). In this study, we produced a highly enriched population of proliferating neural progenitors derived from SDIA-treated monkey ES cells. Furthermore, treatment of these cells with a combination of FGF2 and FGF20 induced the generation of a large

\section{Figure 5}

Survival of ES cell-derived DA neurons in the striatum. (A-C) Grafted cells (BrdU-labeled, green) survived and differentiated into DA neurons (TH-positive, red) along the needle tract (merged image C). Scale bar: $500 \mu \mathrm{m}$. (D-I) Colocalization (arrows in $\mathbf{F}$ and $\mathbf{I}$ ) of BrdU (D, F, G, and $\mathbf{I}$, green) and TH (E and $\mathbf{F}$, red) or DAT (H and $\mathbf{I}$, red) shows that graft-derived cells have dopaminergic character. Scale bar: $50 \mu \mathrm{m}$.
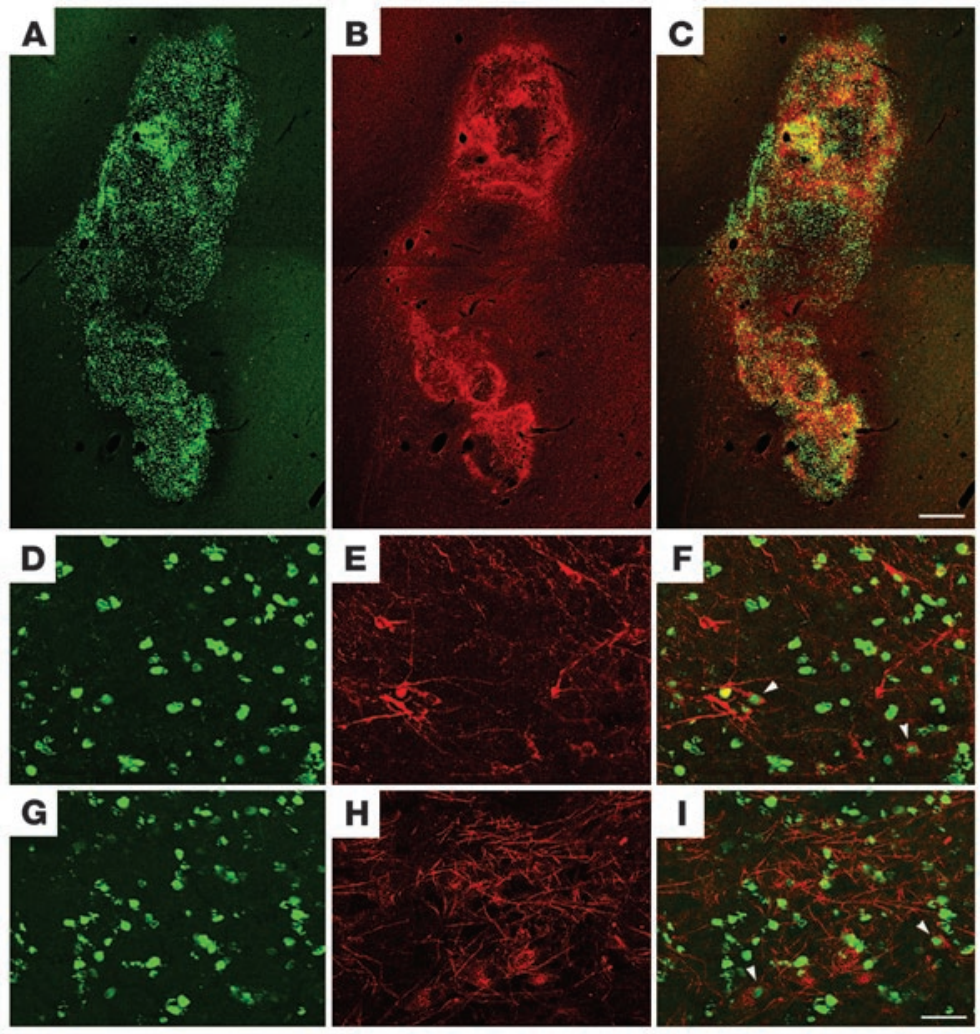
neurospheres was $5.4 \pm 1.8 \%$ of TuJ 1 , much lower than that generated by culturing on PA6 cells (35\%; ref. 10). This discrepancy most likely results from mechanical damage caused by detaching the cells or inappropriate culture conditions for spheres. To increase the number of DA neurons differentiated from neurospheres, we examined the effects of various additional growth factors. Ascorbic acid and Sonic hedgehog, which were used in the 5-step method (20), did not increase the proportion of TH-positive cells (data not shown). In contrast, FGF20 treatment in combination with FGF2 was able to efficiently increase the proportion of TH-positive cells. FGF20 is a secreted protein that is preferentially expressed in the substantia nigra pars compacta of the rat brain (13). The expression profile of FGF20 is quite different from that of other FGF family members, which suggests that FGF20 plays a unique role in the brain. Furthermore, recombinant FGF20 enhances the survival of primary DA neurons (13). FGF receptor-1c, the receptor through which FGF20 activates the mitogen-activated protein kinase pathway, is also preferentially expressed in the substantia nigra pars compacta (24). Our results raise the possibility that FGF20 in combination with FGF2 may support the survival or promote the proliferation of progenitors of DA neurons, resulting in the enrichment of DA progenitor cells in spheres. The mechanism by which this combined stimulation of FGF2 and FGF20 facilitates the production of DA neurons remains to be clarified.

FGF2 and EGF are reported to play different roles in the differentiation of neural precursors. Although FGF2 and EGF promote proliferation of neural precursor cells, the former promotes neuronal differentiation, while the latter induces glial differentiation $(12,25,26)$. They also have different effects on the differentiation of embryoid bodies derived from human ES cells (27). In the present study using neural precursors derived from primate ES cells, FGF2 increased differentiation of ES cells into DA neurons, while EGF suppressed this process even in the presence of FGF2 and FGF20. It is possible that EGF interferes with the differentiation of SDIA-treated spheres into DA neurons directly or indirectly by promoting astroglial induction. Alternatively, EGF may stimulate proliferation or differentiation of a different cell population than that stimulated by FGF2. The differential effects of growth factors present an intriguing topic for future investigation.

In primate studies, functional neuroimaging is a useful tool for in vivo assessment of differentiation, survival, and functional integration of grafted cells. PET imaging of presynaptic targeting reagents such as fluoro-dopa, fluoro-metatyrosine, or $2 \beta$-carbomethoxy-3 $\beta$-4-fluorophenyltropane (CFT) determines whether cells implanted in vivo have the molecular machinery necessary for dopamine synthesis and/or storage (28-30). In this study, we examined the uptake of fluoro-dopa at 14 weeks after transplantation. The significant increase in the mean Ki value in the putamen of ES cell-transplanted animals indicated that the grafted cells functioned as DA neurons. A postmortem examination of the ES cell-transplanted monkey in Figure 4C revealed that more $\mathrm{TH}$-positive cells survived within the right putamen. This finding reflects the correlation of the PET results with the survival of DA neurons. For future studies, detailed analyses using additional tracers, including postsynaptic markers such as fluoro-raclopride, should allow for further understanding of the functional aspects of grafted cells. In this study, we detected significant differences in the mean $\mathrm{Ki}$ values from the entire putamen between the ES cell-transplanted monkeys and the sham-treated control monkeys 3 months after surgery. Widner et al. (8) reported that striatal uptake of fluoro-dopa was unchanged 5 to 6 months postoperatively, but increased markedly at 12 to 13 and 22 to 24 months in patients who received fetal mesencephalic grafts. Freed et al. (4) reported an improved Ki value from the entire putamen 6 months after transplantation. Given the lengthier monitoring periods in these reports, our evaluation of mean Ki values at 3 months may still be premature; further PET studies at later time points may result in even greater changes.

MPTP is a neurotoxin that causes selective destruction of DA neurons in the substantia nigra pars compacta, inducing PD-like symptoms in primates $(31,32)$. Following repetitive intravenous injections of MPTP (approximately $17 \mathrm{mg}$ in total per animal), monkeys stably exhibited PD-like symptoms more than 12 weeks before transplantation surgery. With a blind evaluation based on neurological scores, we detected significant behavioral improvements in the ES cell-transplanted monkeys 10 weeks after transplantation. Recently, 2 double-blind placebo-controlled clinical trials of fetal nigral transplantation $(4,7)$ demonstrated that younger patients and patients with mild symptoms improved after treatment, with behavioral recovery first observed in the 3 to 4 month period following surgery. In this study, posture and motility were the symptoms showing the most marked improvement. These results are comparable with clinical reports $(4,33)$ demonstrating improvements in rigidity and hypokinesia.

PET and immunofluorescence studies demonstrated that a substantial number of the grafted cells survived in the putamen to function as DA neurons. We transplanted 300,000-600,000 cells into each side of the brain in each monkey. The number of surviving cells detected by BrdU staining was approximately 8,000 per side. Thus, the survival rate of the grafted cells was $1.3 \%$ to $2.7 \%$, although the actual value could be higher (discussed below). Through TH staining, the number of surviving DA neurons was shown to be approximately 4,300 per brain. In normal brains, there are no DA neuron cell bodies in the striatum, only fibers. Thus, these TH-positive cells were considered to be derived from the grafted ES cells. While the grafted cells were labeled by BrdU prior to transplantation, only $65 \%$ of these $\mathrm{TH}$-positive cells were immunoreactive for BrdU. This may have resulted from incomplete labeling of the input cells; as the cells were treated with BrdU while being cultured as spheres, the labeling rate was not $100 \%$, but $68.8 \%$. In addition, grafted cells might proliferate in vivo, reducing the concentration of BrdU in the cells. Furthermore, intrinsic striatal TH-positive neurons may be recruited, as reported previously (34), which may explain the observation of a few TH-positive neurons even in control monkeys.

According to an earlier clinical report, the number of TH-positive cells in the postmortem brain of a PD patient was approximately 200,000 (35). In 2 recent double-blind trials, however, the number of surviving TH-positive cells was determined to be 50,000-240,000 $(4,7)$. Given that the volume of the monkey putamen is $10 \%$ of that of the human putamen $(36,37)$, it is likely that the required number of TH-positive cells in the monkey (M. fascicularis) brain is 5,000-24,000. The results of this study remain in keeping with observations made in human patients, suggesting that ES cells are a promising candidate for a donor source for cell transplantation treatment of PD. It should be noted, however, that the MPTP-treated monkey is a model of acute selective nigral destruction whereas human PD patients also experience progressive deterioration and pathological changes of other neural systems $(15,38,39)$. 
Although the results presented here encourage the development of strategies involving ES cell-derived neurons for treatment of neurological diseases, further studies will be needed to address the long-term efficacy and safety of using these cells. For instance, the low survival rate of the grafted cells or neurons is comparable to that noted in previous reports (40). To increase the number of viable DA neurons produced by grafts in vivo, we used DA neuron progenitors in the present study. Multiple-target grafting (41) is also a strategy that should be considered. Notably, we observed a number of GABA-positive cells in the graft, suggesting that other types of neurons and/or glial cells in the graft may contribute to both the differentiation and function of transplanted DA neurons (42). However, the optimal cellular composition of the graft remains to be determined. In addition, while previous studies with rodents have demonstrated that tumor formation can be associated with ES cell grafts (43-45), we did not observe tumor formation or Ki67-positive cells within the first 3 months after transplantation. In the future, however, it will be important to examine late tumor formation as well as the possible long-term effects of ES cell grafts on motor behavior. It will also be necessary to use non-THpositive cells in control grafts to exclude the possibility that the effects of this treatment are mediated by non-DA cells.

Finally, we would like to emphasize that our system (MRI, surgery, PET, etc.) is applicable to humans. Previous work has shown that monkey ES cells have characteristics similar to those of human ES cells $(18,19,46)$. In addition, it was recently demonstrated that neural precursors induced from human ES cells were able to survive in rodent brains $(47,48)$. The SDIA method is applicable to human ES cells, allowing for enrichment of DA progenitors (unpublished data). These results suggest that transplantation using ES cells as a clinical therapy for PD is approaching the point of technical feasibility. Two recent double-blind, sham surgery-controlled trials of embryonic mesencephalic transplants for the treatment of PD, however, showed only modest improvement $(4,7)$, suggesting the potential limits of cell transplantation. Many basic issues, especially regarding stem cell therapy, remain to be resolved (38). Before the clinical application of human ES cell transplantation can be attempted, extensive studies assessing the safety and efficacy of ES cell transplantation in monkey models will be necessary.

\section{Methods}

Maintenance of primate ES cells. Cynomolgus monkey ES cell lines were established and their pluripotency confirmed by teratoma formation in mice with severe combined immunodeficiency, as previously described (19). Undifferentiated ES cells were maintained on a feeder layer (i.e., STO) of embryonic fibroblasts treated with mitomycin C (Wako Pure Chemical Industries Ltd.) in DMEM (Sigma-Aldrich)/F-12 (Invitrogen Corp.) supplemented with $0.1 \mathrm{mM} 2$-mercaptoethanol (2-ME) (SigmaAldrich), 1,000 units/ml LIF (Chemicon International), 20\% knockout serum replacement (KSR; Invitrogen Corp.), and 4 ng/ml FGF2 (Upstate Biotechnology Inc.). ES cells were subcultured using $0.25 \%$ trypsin (Invitrogen Corp.) in PBS with $20 \% \mathrm{KSR}$ and $1 \mathrm{mM} \mathrm{CaCl}_{2}$ (Wako Pure Chemical Industries Ltd.) as described $(11,19)$.

Induction of neural progenitors from primate ES cells. PA6 cells plated on type I collagen-coated chamber slides (BD) or gelatin-coated dishes (gelatin from Sigma-Aldrich; dishes from BD) were used as a feeder cell layer. To avoid contamination by incidentally differentiating cells, we manually selected undifferentiated ES cell colonies with stem cell-like morphology (tightly packed cells exhibiting a high nucleus to cytoplasm ratio). Undifferenti- ated ES cell colonies were washed twice with Glasgow minimum essential medium (GMEM) (Sigma-Aldrich) supplemented with 10\% KSR, $1 \mathrm{mM}$ pyruvate (Sigma-Aldrich), $0.1 \mu \mathrm{M}$ nonessential amino acids, and $0.1 \mathrm{mM}$ 2-ME. After trypsinization, partially dissociated ES cell clumps (10-50 cells per clump) were plated on PA6 cells at 1000 clumps per 10-cm dish. Cells were then cultured in GMEM supplemented with $5 \% \mathrm{KSR}, 2 \mathrm{mM}$ glutamine, $0.1 \mathrm{mM}$ nonessential amino acids, $1 \mathrm{mM}$ pyruvate, and $0.1 \mathrm{mM} 2-\mathrm{ME}$ for 2 weeks. Differentiated colonies were detached from feeder cells using a papain dissociation system (Worthington Biochemical Corp.). Isolated colonies were cultured in neurobasal medium (Invitrogen Corp.) supplemented with B27 supplement (Invitrogen Corp.), $20 \mathrm{ng} / \mathrm{ml} \mathrm{FGF2,} \mathrm{and} 20$ $\mathrm{ng} / \mathrm{ml}$ EGF (R\&D Systems) for 1 week. In the experiments examining the effecs of growth factors on neurosphere culture, the indicated concentrations of FGF20 $(1 \mathrm{pg} / \mathrm{ml}, 10 \mathrm{pg} / \mathrm{ml}$ and $1 \mathrm{ng} / \mathrm{ml})$ were added to medium with or without FGF2 and EGF. In order to evaluate the expression of neural progenitor cell markers by each cell, spheres were incubated with papain at $37^{\circ} \mathrm{C}$ for 10 minutes and then mechanically dissociated into single cells. After incubation with a papain inhibitor, the dissociated cells were plated on OL-coated slides (OL from Sigma-Aldrich) at a density of $10^{5} \mathrm{cells} / \mathrm{cm}^{2}$. After 16 hours, they were fixed in $4 \%$ paraformaldehyde (Sigma-Aldrich) and evaluated by immunofluorescence.

Differentiation of neural progenitor cells. Spheres were isolated manually and plated on OL-coated slides in neurobasal medium to which had been added B27 supplement, $20 \mathrm{ng} / \mathrm{ml}$ brain-derived neurotrophic factor (Sigma-Aldrich), and $20 \mathrm{ng} / \mathrm{ml}$ neurotrophin-3 (Sigma-Aldrich). After 1 week of culture, spheres were fixed or used for additional experiments.

RT-PCR analysis. Total RNA was isolated from differentiated cells using a TRIzol kit (Invitrogen Corp.) according to the manufacturer's instructions. cDNA synthesis was carried out using the SuperScript Double Stranded cDNA Synthesis kit (Invitrogen Corp.). PCR was performed using KODplus polymerase (Toyobo Co.), with the following cycling conditions: 30 seconds at $94^{\circ} \mathrm{C}, 30$ seconds at $60.5^{\circ} \mathrm{C}, 60$ seconds at $68^{\circ} \mathrm{C} \times 30$ cycles for Pax2, Ptx3, Nurr1, and GAPDH; and 30 seconds at $94^{\circ} \mathrm{C}, 30$ seconds at $66^{\circ} \mathrm{C}, 60$ seconds at $68^{\circ} \mathrm{C} \times 30$ cycles for Lmx $1 \mathrm{~b}$ and TH in a thermal cycler (Astec Co.). The experiments were repeated 4 to 7 times for confirmation, and PCR products were sequenced to rule out false positives. The primers used are as follows: Pax2, TGTGTCAGCAAAATCCTGGGCAGGT and TGCTGAACTTTGgtCCGgAtgat; Ptx3, TTCGCCTTCAACTCGGTCAACGT and CCCAGgGTCTGAAAGGGGTG; Nurr1, CTCCCAGAGGGAACTGCACTTCG and CTCTGGAGTTAAGAAATCGGAGCTG; $L m x 1 b$, GCAGCGGCTGCATGGAGAAGATCGC and GGTTCTGAAACCAGACCTGGACCAC; TH, GACTGCTGCCACGAGCTGCTGGG and TCTTGGTAGGGCTGCACGG; and GAPDH, GTGAAGGTCGGAGTCAACG and GGTGAAGACGCCAGTGGACTC.

Dopamine release assay. Spheres treated with FGF2 and FGF20 were plated onto $35 \mathrm{~mm}$ OL-coated dishes at a density of 50 spheres per dish and made to differentiate for 1 week. Then, after being rinsed in a low- $\mathrm{KCl}$ $(4.7 \mathrm{mM})$ solution, the cells were incubated in $1 \mathrm{ml}$ of a high- $\mathrm{KCl}$ solution (20 mM HEPES-NaOH, pH 7.4, 85 mM NaCl, 60 mM KCl, $2.5 \mathrm{mM} \mathrm{CaCl}_{2}$, $1.2 \mathrm{mM} \mathrm{MgSO}_{4}, 1.2 \mathrm{mM} \mathrm{KH}_{2} \mathrm{PO}_{4}$, and $11 \mathrm{mM}$ glucose) for 15 minutes. Concentrations of dopamine were determined by HPLC using a reversephase column and an electrochemical detector system (HTEC 500; Eicom Corp.) as previously described (49).

Animal model. Adult male cynomolgus monkeys (M. fascicularis) weighing $2.5-3.5 \mathrm{~kg}$ were given intravenous injections of MPTP $\mathrm{HCl}(0.4 \mathrm{mg} / \mathrm{kg}$ as free base, Sigma-Aldrich) twice a week until persistent Parkinsonian behavioral disturbances, such as tremor, bradykinesia, and impaired balance, became evident. Animals were given an average of 14.7 MPTP shots and exhibited stable Parkinsonism for approximately 30 days. To prevent any possibility of spontaneous recovery, only those monkeys that presented 
stable deterioration for a period greater than 12 weeks were used for transplantation experiments. All animals were fed with commercial pellets and fresh fruits and had free access to clean water. Monkeys were cared for and handled according to Guidelines for Animal Experiments of Kyoto University and the National Cardiovascular Center (Osaka, Japan) and the Guide for the Care and Use of Laboratory Animals from the Institute of Laboratory Animal Resources (ILAR; Washington, DC, USA).

MRI. For accurate orientation of the putamen, animals $(n=10 ; 6$ for ES transplanted and 4 for sham-operated group) were subjected to MRI examination using a 3.0 Tesla Signa system (General Electric). Following anesthesia by intramuscular injection with ketamine hydrochloride $(15 \mathrm{mg} / \mathrm{kg}$; Sankyo Co.) and xylazine $(1.5 \mathrm{mg} / \mathrm{kg}$; Boehringer Ingelheim Vetmedica Inc.), animals were positioned into the magnet using an MR-compatible headholder. T1-weighted images were used for further examination.

Transplantation. Following anesthesia with pentobarbital $(7.5 \mathrm{mg} / \mathrm{kg}$, intramuscularly [i.m.]; Dainippon Pharmaceutical Co.) and ketamine (10 $\mathrm{mg} / \mathrm{kg}, \mathrm{i} . \mathrm{m}$.), monkeys were fixed in a surgical frame (Narishige SN-1N; Narishige Co.). Neural progenitors from monkey ES cell cultures in two 6-cm dishes (300,000-600,000 cells per dish) were collected for each animal. While the ES cells were expanding as spheres, BrdU (5 $\mu \mathrm{g} / \mathrm{ml}$; SigmaAldrich) was added to the culture medium for 7 days to label the ES cells. Using an electric injector (Muromachi Kikai Co.), we transplanted donor cells into the putamen bilaterally, using the MRI findings for each monkey and the M. fascicularis brain atlas $(36,50)$. To cover the mid to posterior putamen, 3 targets that were $2 \mathrm{~mm}$ apart in anterior-posterior position and $1-2 \mathrm{~mm}$ in medial-lateral position were set, and 4 injections $(1 \mu \mathrm{l} / 60$ seconds for each injection) were made along each tract. After surgery, all animals were given antibiotics for 1 week and a daily immunosuppressant (cyclosporin A, $10 \mathrm{mg} / \mathrm{kg}$, i.m.; Carbiochem) until sacrifice. Monthly blood analyses performed over the course of the experiment confirmed the levels of circulating cyclosporin A, which averaged $393 \mathrm{ng} / \mathrm{ml}$. In control monkeys undergoing sham operations, the equivalent volume of culture medium was administered without cells. Afterward, antibiotics and immunosuppressants were injected as for the cell-grafted monkeys.

Immunofluoresence study. For in vitro studies, cells were fixed in $4 \%$ paraformaldehyde for 10 minutes for microscopic observation. For in vivo studies, after administration of deep anesthesia with pentobarbital and ketamine, monkeys ( $n=10 ; 6$ for ES cell-transplanted and 4 for shamoperated group) were transcardially perfused with $4 \%$ paraformaldehyde. Excised brains were frozen and cut with a microtome at a $50-\mu \mathrm{m}$ thickness. Sections were then stained by the free-floating method. Slides were first incubated in $0.3 \%$ Triton $\mathrm{X}$ with $5 \%$ skim milk in PBS for 30 minutes. Samples were then incubated with one of the following primary antibodies: rabbit anti-NCAM (1:300; Chemicon Inc.), rat anti-Musashi-1 (1:200; gift from H. Okano, Keio University, Japan), mouse anti-Nestin (1:300; Chemicon Inc.), mouse anti-TuJ1 (1:300; BabCO), rabbit anti-GFAP (1:1000; Chemicon Inc.), mouse anti-Map2ab (1:250; Sigma-Aldrich), rabbit anti-GalC (1:100; Chemicon Inc.), rabbit anti-glutamate (1:200; Chemicon Inc.), goat anti-ChAT (1:1000; Chemicon Inc.), mouse antiGABA (1:1000; Sigma-Aldrich), mouse anti-BrdU (1:200; BD), rabbit anti-TH (1:60; Chemicon Inc.), rabbit anti-serotonin (1:2000; DiaSorin Inc.), or rat anti-DAT (1:100; Chemicon Inc.) in $2 \%$ skim milk in PBS overnight at $4^{\circ} \mathrm{C}$. After 3 washes in PBS, samples were incubated with the appropriate secondary antibodies for 1 hour at room temperature: FITC-labeled anti-mouse Ig (1:200; Jackson Immunoresearch Laboratories Inc.) and Cy3-labeled anti-rabbit Ig (1:200; Jackson Immunoresearch Laboratories Inc.). For nuclear staining, $200 \mathrm{ng} / \mathrm{ml}$ of 4',6'-diamidino-2phenylindole (DAPI) was added in the final wash. After being washed in PBS, samples were mounted and analyzed using a Fluoview FV300 laser confocal microscope (Olympus Optical Co.). For in vitro studies, cells in
5 randomly selected fields, each of which included 100-500 cells, were counted for 3 to 5 independent cultures. For in vivo studies, the number of immunoreactive cells was quantified in every sixth section throughout the graft and surrounding tissue. Statistical analysis was performed by one-way ANOVA and post hoc multiple comparison by the Dunn test. A $P$ value of less than 0.05 was considered significant.

Behavioral assessment. Parkinsonian behavior was evaluated using a rating scale previously proposed by Akai et al. (51), with modifications (Table 1). To prevent subjective biases, the evaluation was performed by a trained examiner who was not involved in the culturing and transplantation of the cells and not informed of the specific procedure to which each monkey was subjected. Student's $t$ test was used to compare the 2 groups, and a $P$ value of less than 0.05 was considered significant.

$P E T$. To evaluate the in vivo DA function of the grafted cells, PET scans using ${ }^{18} \mathrm{~F}$-fluorodopa were performed on each animal prior to perfusion. Under generalized anesthesia with a continuous infusion of propofol (4 mg/kg/h; Zeneca Pharmaceuticals) and vecuronium-bromide (0.25 $\mathrm{mg} / \mathrm{kg} / \mathrm{h}$; Boehringer Ingelheim Vetmedica Inc.), analyses were performed using an ECAT EXACT HR+ PET scanner (Siemens/CTI). After intravenous injection of $185 \mathrm{MBq}$ of ${ }^{18} \mathrm{~F}$-fluorodopa, brain radioactivity was assessed for 90 minutes in animals that had received carbidopa $(10 \mathrm{mg} / \mathrm{kg}) 30 \mathrm{~min}-$ utes prior to the PET scan. Parametric images of the dopamine-irreversible metabolic rate of $\mathrm{Ki}\left(\mathrm{min}^{-1}\right)$, considered to be a measure of presynaptic DA function, were generated using the time course of radioactivity in each voxel by multiple-time graphical analysis (52) using the bilateral occipital lobes as a reference region. The ${ }^{18} \mathrm{~F}$-fluorodopa $\mathrm{Ki}$ image was coregistered to the corresponding T1-weighted MRI image, which was obtained by an inversion-recovery prepared fast spoiled gradient recalled acquisition in the steady state (IR-FSPGR) sequence $(\mathrm{Tr}=9.4, \mathrm{TI}=600, \mathrm{TE}=2.1$ in $\mathrm{msec}$ ) using a 3.0 Tesla Signa System (General Electric) and realigned to a standard space of $M$. fascicularis $(36,50)$. DA function was evaluated by visual inspection of $\mathrm{Ki}$ images and by quantitative Ki values in the bilateral striatum identified by the corresponding MRI image. This evaluation was performed in a blind manner to ensure objectivity. Student's $t$ test was used to compare the results.

\section{Acknowledgments}

The authors thank H. Sameshima and T. Yamamoto (Shin Nippon Biomedical Laboratories Ltd.) for technical help with monkeys, H. Okano (Keio University) for kindly providing the Musashi-1 antibody, and Hiroyuki Nawa (Niigata University) for helpful advice regarding the dopamine release assay. We also thank Y. Yanagi and T. Gomibuchi for their secretarial help. This study was supported by the following: Grants-in-Aid for Scientific Research, Grants in Kobe Knowledge Cluster, and Establishment of International Center of Excellence for Integration of Transplantation Therapy and Regenerative Medicine from the Ministry of Education, Culture, Sports, Science and Technology; Health Sciences Research Grants in Research on Human Genome, Tissue Engineering and Food Biotechnology from the Ministry of Health, Labour and Welfare; and Grants in Organization for Pharmaceutical Safety and Research.

Received for publication January 22, 2004, and accepted in revised form November 2, 2004.

Address correspondence to: Jun Takahashi, Department of Neurosurgery, Kyoto University Graduate School of Medicine, 54 Kawahara-cho, Shogoin, Sakyo, Kyoto 606-8507, Japan. Phone: 81-75-7513450; Fax: 81-75-752-9501; E-mail: jbtaka@kuhp.kyoto-u.ac.jp. 
1. Bergman, H., and Deuschl, G. 2002. Pathophysiology of Parkinson's disease: from clinical neurology to basic neuroscience and back. Mov Disord. 17(Suppl. 3):S28-S40.

2. Dostrovsky, J.O., Hutchison, W.D., and Lozano, A.M. 2002. The globus pallidus, deep brain stimulation, and Parkinson's disease. Neuroscientist. 8:284-290.

3. Miyasaki, J.M., Martin, W., Suchowersky, O., Weiner, W.J., and Lang, A.E. 2002. Practice parameter: initiation of treatment for Parkinson's disease: an evidence-based review: report of the Quality Standards Subcommittee of the American Academy of Neurology. Neurology. 58:11-17.

4. Freed, C.R., et al. 2001. Transplantation of embryonic dopamine neurons for severe Parkinson's disease. N. Engl. J. Med. 344:710-719.

5. Hagell, P., and Brundin, P. 2001. Cell survival and clinical outcome following intrastriatal transplantation in Parkinson disease. J. Neuropathol. Exp. Neurol. 60:741-752.

6. Lindvall, O., et al. 1994. Evidence for long-term survival and function of dopaminergic grafts in progressive Parkinson's disease. Ann. Neurol. 35: $172-180$.

7. Olanow, C.W., et al. 2003. A double-blind controlled trial of bilateral fetal nigral transplantation in Parkinson's disease. Ann. Neurol. 54:403-414.

8. Widner, H., et al. 1992. Bilateral fetal mesencephalic grafting in two patients with parkinsonism induced by 1-methyl-4-phenyl-1,2,3,6-tetrahydropyridine (MPTP). N. Engl. J. Med. 327:1556-1563.

9. Smith, A.G. 2001. Embryo-derived stem cells of mice and men. Annu. Rev. Cell Dev. Biol. 17:435-462.

10. Kawasaki, H., et al. 2000. Induction of midbrain dopaminergic neurons from ES cells by stromal cell-derived inducing activity. Neuron. 28:31-40.

11. Kawasaki, H., et al. 2002. Generation of dopaminergic neurons and pigmented epithelia from primate ES cells by stromal cell-derived inducing activity. Proc. Natl. Acad. Sci. U. S. A. 99:1580-1585.

12. Gage, F.H. 2000. Mammalian neural stem cells. Science. 287:1433-1438.

13. Ohmachi, S., et al. 2000. FGF-20, a novel neurotrophic factor, preferentially expressed in the substantia nigra pars compacta of rat brain. Biochem. Biophys. Res. Commun. 277:355-360.

14. Tonchev, A.B., Yamashima, T., Zhao, L., Okano, H.J., and Okano, H. 2003. Proliferation of neural and neuronal progenitors after global brain ischemia in young adult macaque monkeys. $\mathrm{Mol}$. Cell. Neurosci. 23:292-301.

15. Arenas, E. 2002. Stem cells in the treatment of Parkinson's disease. Brain Res. Bull. 57:795-808.

16. Smidt, M.P., et al. 2000. A second independent pathway for development of mesencephalic dopaminergic neurons requires $L m x 1 b$. Nat. Neurosci. 3:337-341.

17. Ranjita, B., et al. 1997. Dopaminergic neurons intrinsic to the primate striatum. J. Neurosci. 17:6761-6768.

18. Thomson, J.A., et al. 1995. Isolation of a primate embryonic stem cell line. Proc. Natl. Acad. Sci. U.S. A. 92:7844-7848

19. Suemori, H., et al. 2001. Establishment of embryonic stem cell lines from cynomolgus monkey blastocysts produced by IVF or ICSI. Dev. Dyn.
222:273-279.

20. Lee, S.H., Lumelsky, N., Studer, L., Auerbach, J.M., and McKay, R.D. 2000. Efficient generation of midbrain and hindbrain neurons from mouse embryonic stem cells. Nat. Biotechnol. 18:675-679.

21. Kim, J.H., et al. 2002. Dopamine neurons derived from embryonic stem cells function in an animal model of Parkinson's disease. Nature. 418:50-56.

22. Morizane, A., Takahashi, J., Takagi, Y., Sasai, Y., and Hashimoto, N. 2002. Optimal conditions for in vivo induction of dopaminergic neurons from ES cells through stromal cell-derived inducing activity. J. Neurosci. Res. 69:934-939.

23. Elsworth, J.D., et al. 1996. Early gestational mesencephalon grafts, but not later gestational mesencephalon, cerebellum or sham grafts, increase dopamine in caudate nucleus of MPTP-treated monkeys. Neuroscience. 72:477-484.

24. Ohmachi, S., Mikami, T., Konishi, M., Miyake, A., and Ito, N. 2003. Preferential neurotrophic activity of fibroblast growth factor-20 for dopaminergic neurons through fibroblast growth factor receptor-1c. J. Neurosci. Res. 72:436-443.

25. Ciccolini, F., and Svendsen, C.N. 1998. Fibroblast growth factor 2 (FGF-2) promotes acquisition of epidermal growth factor (EGF) responsiveness in mouse striatal precursor cells: identification of neural precursors responding to both EGF and FGF-2. J. Neurosci. 18:7869-7880.

26. Kuhn, H.G., Winkler, J., Kempermann, G., Thal, L.J., and Gage, F.H. 1997. Epidermal growth factor and fibroblast growth factor- 2 have different effects on neural progenitors in the adult rat brain. J. Neurosci. 17:5820-5829.

27. Schuldiner, M., Tanuka, O., Itskovitz-Eldor, J., Melton, D.A., and Benvenisty, N. 2000. Effects of eight growth factors on the differentiation of cells derived from human embryonic stem cells. Proc. Natl. Acad. Sci. U. S. A. 97:11307-11312.

28. Doudet, D.J., et al. 1998. 6-[18F]Fluoro-L-DOPA PET studies of the turnover of dopamine in MPTP-induced parkinsonism in monkeys. Synapse. 29:225-232.

29. Elsworth, J.D., et al. 1994. Novel radioligands for the dopamine transporter demonstrate the presence of intrastriatal nigral grafts in the MPTPtreated monkey: correlation with improved behavioral function. Exp. Neurol. 126:300-304.

30. Poyot, T., et al. 2001. Anatomic and biochemical correlates of the dopamine transporter ligand 11CPE2I in normal and parkinsonian primates: comparison with 6-[18F]fluoro-L-dopa. J. Cereb. Blood Flow Metab. 21:782-792.

31. Bankiewicz, K.S., et al. 1986. Hemiparkinsonism in monkeys after unilateral internal carotid artery infusion of 1-methyl-4-phenyl-1,2,3,6-tetrahydropyridine (MPTP). Life Sci. 39:7-16.

32. Burns, R.S., et al. 1983. A primate model of parkinsonism: selective destruction of dopaminergic neurons in the pars compacta of the substantia nigra by N-methyl-4-phenyl-1,2,3,6-tetrahydropyridine. Proc. Natl. Acad. Sci. U. S. A. 80:4546-4550.

33. Wenning, G.K., et al. 1997. Short- and long-term survival and function of unilateral intrastriatal dopaminergic grafts in Parkinson's disease. Ann. Neurol. 42:95-107.

34. Betarbet, R., et al. 1997. Dopaminergic neurons intrinsic to the primate striatum. J. Neurosci. 17:6761-6768.

35. Kordower, J.H., et al. 1996. Functional fetal nigral grafts in a patient with Parkinson's disease: chemoanatomic, ultrastructural, and metabolic studies. J. Comp. Neurol. 370:203-230.

36. Martin, R.F., and Bowden, D.M. 2000. Primate brain maps: structure of the macaque brain. Volume 8 . Elsevier. New York, New York, USA. 160 pp.

37. Schaltenbrand, G., and Wahren, W. 1977. Atlas for stereotaxy of the human brain. Georg Thieme Verlag. Stuttgart, Germany.

38. Björklund, A., et al. 2003. Neural transplantation for the treatment of Parkinson's disease. Lancet Neurol. 2:437-445.

39. Jenner, P. 2003. The contribution of the MPTPtreated primate model to the development of new treatment strategies for Parkinson's disease. Parkinsonism. Relat. Disord. 9:131-137.

40. Isacson, O., et al. 2001. Cell implantation therapies for Parkinson's disease using neural stem, transgenic or xenogeneic donor cells. Parkinsonism. Relat. Disord. 104:205-212.

41. Collier, T.J., et al. 2002. Embryonic ventral mesencephalic grafts to the substantia nigra of MPTPtreated monkeys: feasibility relevant to multipletarget grafting as a therapy for Parkinson's disease. J. Comp. Neurol. 442:320-330.

42. Björklund, A., and Lindvall, O. 2000. Cell replacement therapies for central nerovous system disorders. Nat. Neurosci. 3:537-544.

43. Björklund, L.M., et al. 2002. Embryonic stem cells develop into functional dopaminergic neurons after transplantation in a Parkinson rat model. Proc. Natl. Acad. Sci. U. S. A. 99:2344-2349.

44. Erdö, F., et al. 2003. Host-dependent tumorigenesis of embryonic stem cell transplantation in experimental stroke. J. Cereb. Blood Flow Metab. 23:780-785.

45. Harkany, T., et al. 2004. Region-specific generation of functional neurons from naive embryonic stem cells in adult brain. J. Neurochem. 88:1229-1239.

46. Thomson, J.A., et al. 1998. Embryonic stem cell lines derived from human blastocysts. Science. 282: $1145-1147$.

47. Reubinoff, B.E., et al. 2001. Neural progenitors from human embryonic stem cells. Nat. Biotechnol. 19:1134-1140.

48. Zhang, S.C., Wernig, M., Duncan, I.D., Brustle, O., and Thomson, J.A. 2001. In vitro differentiation of transplantable neural precursors from human embryonic stem cells. Nat. Biotechnol. 19:1129-1133.

49. Nagano, Y., et al. 2003. Siah-1 facilitates ubiquitination and degradation of Synphilin-1. J. Biol. Chem. 278:51504-51514.

50. Sabo, J., and Cowan, W.M. 1984. A stereotaxic atlas of the brain of the cynomolgus monkey (Macaca fascicularis). J. Comp. Neurol. 222:265-300.

51. Akai, T., Ozawa, M., Yamaguchi, M., Mizuta, E., and Kuno, S. 1995. Combination treatment of the partial D2 agonist terguride with the D1 agonist SKF 82958 in 1-methyl-4-phenyl-1,2,3,6-tetrahydropyridine-lesioned parkinsonian cynomolgus monkeys. J. Pharmacol. Exp. Ther. 273:309-314.

52. Patlak, C.S., and Blasberg, R.G. 1985. Graphical evaluation of blood-to-brain transfer constants from multiple-time uptake data. J. Cereb. Blood Flow Metab. 5:584-590. 